\title{
Calibration of CORSIM Models Considering all Model Parameters Simultaneously*
}

\author{
A. Paz, V. Molano and C. Gaviria
}

This study proposes a calibration methodology for microscopic traffic flow simulation models that has the capability to simultaneously consider all model parameters and also to calibrate such time-dependent aspects of the model as link counts. The Simultaneous Perturbation Stochastic Approximation algorithm provides the optimization engine that determines the calibrated set of model parameters. In this study, experiments were conducted using two different CORSIM models; the results illustrate the effectiveness of the proposed calibration methodology. Current research focuses on expanding the proposed methodology to enable the simultaneous calibration of link counts, speeds, and associated bottlenecks.

\section{INTRODUCTION}

This study seeks to develop a generalized methodology for the calibration of microscopic traffic flow simulation models. The methodology has been developed to simultaneously calibrate all model parameters at a low or reasonable associated cost. This is a desirable characteristic of a generalized calibration methodology. The simultaneous consideration of all model parameters contributes towards the convergence of the optimization algorithm that is required for calibration.

At this point in the model development, the proposed methodology minimizes the difference between actual and simulated time-dependent link counts by considering simultaneously all global and independent link model parameters. The proposed calibration methodology is being developed independent of the characteristics of any particular microscopic traffic flow simulation model. Currently, it is being tested using CORSIM models; further research will test the methodology using various microscopic traffic flow simulation models.

CORSIM has been one of the most popular microscopic traffic flow simulation models on the market. There is a vast number of vehicular traffic systems modeled using CORSIM [1-3]. Many agencies use these models to evaluate potential solutions to emerging traffic problems. In addition, many

\footnotetext{
* Research Supported by Nevada Department of Transportation Dr. Alexander Paz is an Assistant Professor of Civil Engineering at the University of Nevada, Las Vegas, NV 89119 USA (apaz@unlv.edu)

Victor Molano is a graduate student at the University of Nevada-Las Vegas, Las Vegas, NV 89119 USA (molanopa@unlv.nevada.edu)

Carlos Gaviria is an undergraduate student at the University of Cauca, Popayan, Cauca, Colombia (cfgaviria@unicauca.edu.co)
}

traffic professionals have been trained and are very comfortable using CORSIM.

Many of the fundamental problems faced while developing microscopic traffic flow simulation models are common across frameworks. Most agencies use heuristic approaches, which typically require a time-consuming sequential process involving constant user intervention [4]. Deterministic approaches such as the sequential simplex algorithm [5] have been used for the calibration of congested regions. Genetic algorithms $[6,7,8]$ have been also used for calibration and optimization of traffic controls as well as for the location of sensors. Stochastic approximation algorithms $[9,10-12]$ have been used for calibration and optimization of traffic flow model characteristics. Even though calibration approaches have been developed in the past for CORSIM models, there is not yet a comprehensive approach that considers all model parameters with none or minimal user intervention.

The proposed calibration methodology is based on the Simultaneous Perturbation Stochastic Approximation (SPSA) algorithm [13, 14], which has been proposed in the past for the calibration of microscopic traffic flow simulation models [11, 12, 15-18]. Vehicular traffic systems modeled using microscopic traffic flow simulation models typically are characterized by a large number of parameters and stochasticities. These characteristics make the SPSA algorithm a very attractive methodological engine for the calibration of microscopic traffic flow simulation models. Advantages of SPSA over other stochastic approximation approaches are its low computational requirements and its fast convergence $[14,19-21]$.

\section{Methodology}

This methodology calibrates time-dependent link counts by simultaneously adjusting all the parameters $\theta$ of the microscopic traffic flow simulation model. Microscopic traffic flow simulation models use a vast number of model parameters -- ranging from driver behavior to vehicle performance -- that have various effects on model performance $[3,4,22-25]$. Hence, it is important to simultaneously consider all model parameters with the aim to capture their intricate effects, thereby enabling convergence.

A mathematical programming approach is used to formulate this calibration problem. The analysis period is divided into a number $T$ of discrete time periods. The objective function, denoted by Equation (1), is the sum over all calibration time-periods $T$ of the root mean square (RMS) of the sum over all links $I$ of the square of the differences 
between actual (V) and simulated ( $\tilde{V})$ link counts. The calibration problem is formulated as follows:

$$
\text { Minimize } R M S=\sum_{t=1}^{T} \sqrt{\sum_{i=1}^{I}\left(V_{i}-\tilde{V}(\theta)_{i}\right)^{2}}
$$

Subject to:

Lower bound $\leq \theta \leq$ Upper bound

A broad number of optimization algorithms, ranging from genetic algorithms to finite difference stochastic approximation, can be used to determine the optimal vector of model parameters $\theta^{*}[5,6,22,24,26]$. In this study, SPSA is used because it only requires two traffic flow simulation runs to update all model parameters per iteration during the search for $\theta^{*}$. Running a low number of traffic flow simulations represents a low computational burden and associated time savings.

\section{CAlibRATION CRITERIA}

The calibration criteria for this study are based on guidelines from the Federal Highway Administration. Table I summarizes these guidelines [23].

TABLE I. CALIBRATION GUIDELINES FOR MICROSCOPIC TRAFFIC FLOW SIMULATION MODELS

\begin{tabular}{|c|l|c|l|}
\hline \multirow{2}{*}{$\begin{array}{c}\text { Traffic } \\
\text { Volumes }\end{array}$} & $\begin{array}{l}\text { Difference between actual } \\
\text { and simulated link counts }\end{array}$ & $<5 \%$ & For all links \\
\cline { 2 - 4 } & GEH statistic & $<5$ & $\begin{array}{l}\text { For at least } \\
85 \% \text { of the } \\
\text { links }\end{array}$ \\
\cline { 2 - 4 } & $\begin{array}{l}\text { Difference between actual } \\
\text { and simulated link counts } \\
\text { for links with more than } \\
8000 \text { vph }\end{array}$ & $\begin{array}{l}\text { Difference } \\
<400 \mathrm{vph} .\end{array}$ & $\begin{array}{l}\text { For at least } \\
\text { links }\end{array}$ \\
\hline
\end{tabular}

$$
\mathrm{GEH}=\sqrt{\frac{2\left(V_{i}-\tilde{V}(\theta)_{i}\right)^{2}}{V_{i}+\tilde{V}(\theta)_{i}}}
$$

where,

$V_{i}=$ actual link counts

$\tilde{\mathrm{V}}(\theta)_{i}=$ simulated link counts

\section{SimultaneOUS PERTURBATION STOCHASTIC APPROXIMATION}

The SPSA algorithm is an iterative approach that uses gradient estimations of the objective function to determine an optimal solution. Details of its implementation are provided by James C. Spall $[13,14]$. In each iteration of SPSA, the vector of model parameters is updated using Equation (4).

$\theta_{k+1}=\theta_{k}-a_{k} g_{k} \theta_{k}$ where,

$\theta_{k+1}=$ vector of updated parameters at iteration $k+1$

$\theta_{k}=\quad$ vector of initial parameters at iteration $k+1$

$a_{k}=\quad$ gain coefficient at iteration $k+1$ calculated using Equation (5)

$g_{k} \theta_{k}=$ estimated gradient at iteration $k+1$.

$a_{k}=\frac{a}{(k+1+A)^{\alpha}}$

where $a, A$, and $\alpha$ are empirical non-negative coefficients. These coefficients affect the convergence of the SPSA algorithm. The simultaneous perturbation and gradient estimate are represented by $g_{k} \theta_{k}$, and is calculated using Equation (6).

$g_{k} \theta_{k}=\frac{y\left(\theta_{k}+c_{k} \Delta_{k}\right)-y\left(\theta_{k}-c_{k} \Delta_{k}\right)}{2 c k}\left[\Delta_{k 1}^{-1}, \Delta_{k 2}^{-1}, \Delta_{k 3}^{-1}, \ldots, \Delta_{k p}^{-1}\right]^{T}$

Here, $c_{k}$ is calculated using Equation (7) where $c$ and $Y$ are empirical non negative coefficients.

$c_{k}=\frac{c}{(k+1)^{r}}$

The elements in the random perturbation vector $\Delta_{k}=$ $\left[\Delta_{k 1}^{-1}, \Delta_{k 2}^{-1}, \Delta_{k 3}^{-1}, \ldots, \Delta_{k p}^{-1}\right]^{T}$ are Bernoulli-distributed, with a probability of one-half for each of the two possible outcomes.

The SPSA algorithm is implemented using the following steps [14]:

Step 1: Set counter $k$ equal to zero. Initialization of coefficients for the gain function $a, A$, and $\alpha$ and calibration parameters $\theta_{0}$.

Step 2: Generation of the random perturbation vector $\Delta_{k}$.

Step 3: Evaluation of the objective function plus and minus the perturbation.

Step 4: Evaluation of the gradient approximation $\mathrm{g}_{k} \theta_{k}$.

Step 5: Update the vector of calibration parameters using Equation (3) along with the corresponding constraints denoted by Equation (2).

Step 6: Check for convergence. If convergence is achieved, stop; otherwise, set counter $k=k+1$ and repeat Steps 1-6. Convergence is achieved when all the criteria in Table I is satisfied or the maximum number of iterations is reached.

\section{EXPERIMENTS AND RESULTS}

Two experiments were conducted to test the effectiveness of the proposed calibration framework. The first experiment calibrated a network with 38 links and a simulation time period of 150 minutes. The second experiment calibrated a network with 20 links and four time-periods of 15 minutes of simulation each for a total of one hour. Hence, the second experiment calibrated time-dependent link counts. The coefficients of the SPSA algorithm were $a=0.002, A=15, \alpha$ 
$=0.602, \mathrm{c}=0.101$, and $Y=0.05$ [14]. These values are recommended in the literature. They affect the convergence of the algorithm. Sensitivity analysis and a bi-level optimization approach currently are being conducted to determine optimal values for these coefficients.

\section{A. Results from the First Experiment}

Fig 1. Illustrates how the objective function is minimized, using the algorithm proposed in Section IV. The noisy trajectory is a consequence of the stochastic perturbation applied to all calibration parameters at each iteration. The RSM is 441.57 before calibration and 12 after calibration.

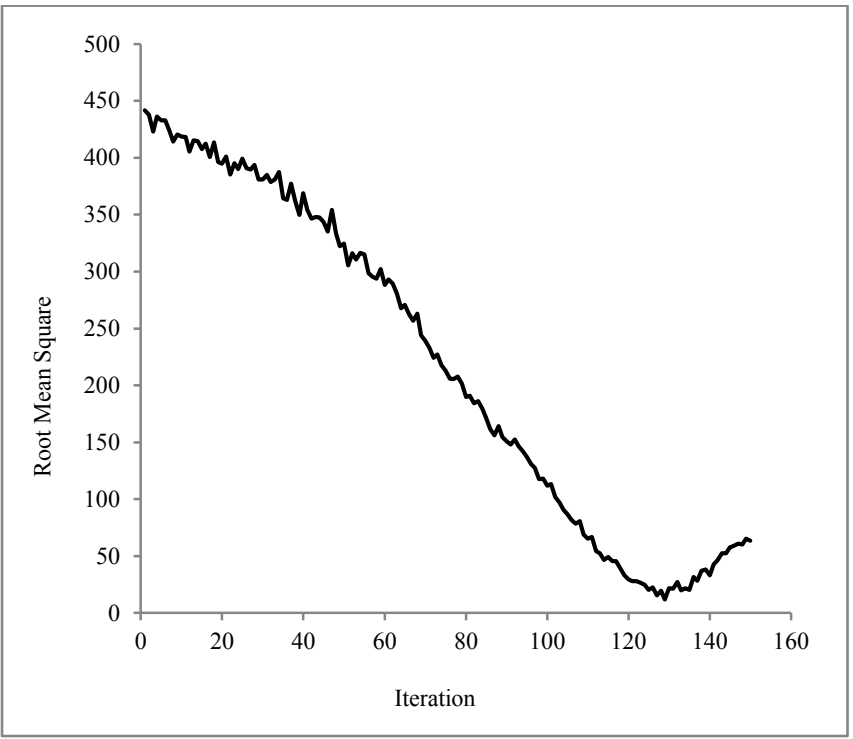

Figure 1. Objective function for the first experiment.

Fig. 2 shows the actual, initial simulated, and calibrated link counts for all links in the network of the first experiment. The dashed line, illustrating the calibrated values, depicts a close approximation to the actual values.

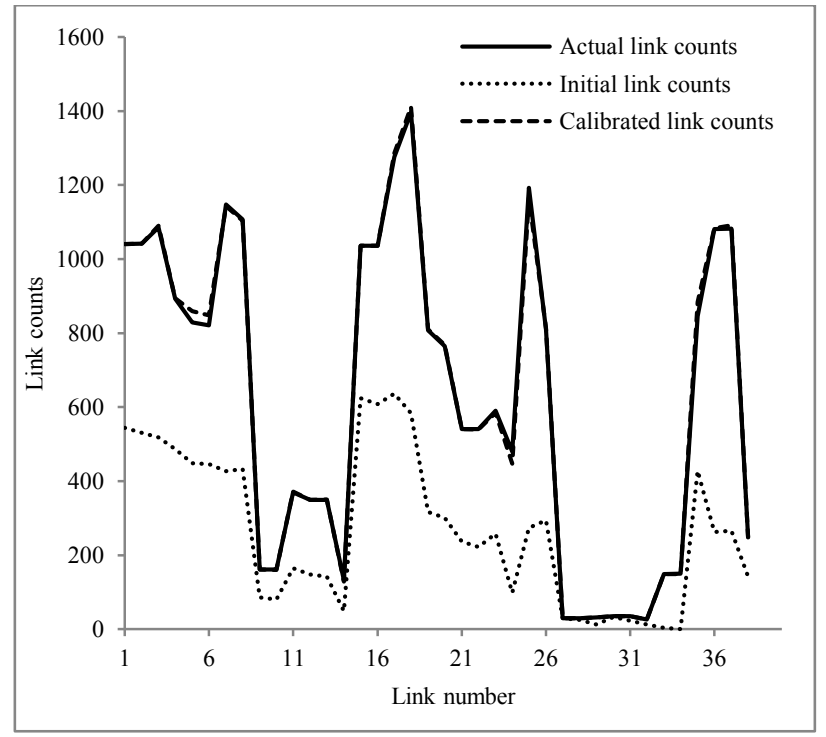

Figure 2. Link counts for the first experiment.
Fig. 3 shows the GEH statistics for the initial (dotted) and calibrated (solid) models. It is clear that the calibration model significantly improves the GEH statistic. All the links reach a GEH statistic less or equal to 5, thereby satisfying the calibration criteria.

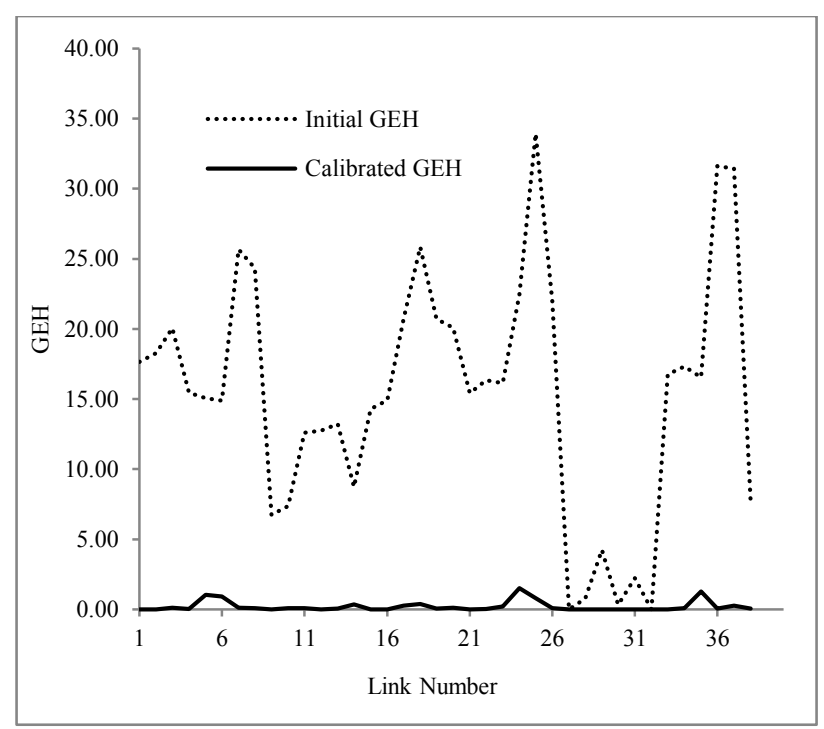

Figure 3. GEH Statistic for the first experiment.

The results show that the three calibration criteria are satisfied. Table II summarizes the calibration results for the first experiment. The objective function improves from 441.57 to 12 . The difference between actual and simulated link counts is less than 5\% for all links in the network, and the GEH statistic is less than 5 for $100 \%$ of the links.

TABLE II. SUMMARY OF CALIBRATION RESUltS FOR THE FIRST EXPERIMENT

\begin{tabular}{|l|c|c|c|}
\hline & RMS & $\begin{array}{c}\text { Total link } \\
\text { counts }\end{array}$ & GEH \\
\hline Initial & 441.57 & 10189 & $<5$ for $15.8 \%$ of the cases \\
\hline Calibrated & 12 & 23769 & $<5$ for all cases \\
\hline Actual & & 2703 & \\
\hline
\end{tabular}

\section{B. Results for the Second Experiment}

Fig. 4 illustrates the trajectory of the objective function corresponding to the second experiment. The initial RMS value is 464.65 while the minimum obtained after 150 iterations of the optimization algorithm is 34.34 .

In this experiment, all parameters for all links for all four time periods were updated. The total time of the simulation is one hour and is divided into time intervals of 15 minutes. The traffic flow rates of vehicles and the percentage of trucks entering the network are variable across time intervals. Table II shows these time-dependent flow rates and the corresponding percentage of trucks entering the network through six loading links.

Fig. 5 and Fig 6, respectively, illustrate the link counts and GEH statistics results for all links in the network for the first time period of the simulation. These results clearly show that the calibration process significantly reduces the difference between actual and simulated link counts. 
TABLE III. Flow RAtes AND PeRCENTAge of TRUCKS ENTERING THE NETWORK

\begin{tabular}{|c|c|c|c|c|c|c|c|}
\hline \multicolumn{2}{|c|}{ Time period 1 } & \multicolumn{2}{c|}{ Time period 2 } & \multicolumn{2}{c|}{ Time period 3 } & \multicolumn{2}{c|}{ Time period 4 } \\
\hline $\begin{array}{c}\text { Flow } \\
\text { rate } \\
\text { (vph) }\end{array}$ & $\begin{array}{c}\text { \% of } \\
\text { trucks }\end{array}$ & $\begin{array}{c}\text { Flow } \\
\text { rate } \\
\text { (vph) }\end{array}$ & $\begin{array}{c}\text { \% of } \\
\text { trucks }\end{array}$ & $\begin{array}{c}\text { Flow } \\
\text { rate } \\
\text { (vph) }\end{array}$ & $\begin{array}{c}\text { \%of } \\
\text { trucks }\end{array}$ & $\begin{array}{c}\text { Flow } \\
\text { rate } \\
\text { (vph) }\end{array}$ & $\begin{array}{c}\% \text { of } \\
\text { trucks }\end{array}$ \\
\hline 1042 & 3 & 1228 & 4 & 1436 & 6 & 1484 & 3 \\
\hline 162 & 0 & 248 & 0 & 284 & 0 & 252 & 0 \\
\hline 350 & 0 & 504 & 0 & 572 & 0 & 620 & 0 \\
\hline 1037 & 5 & 1296 & 6 & 344 & 6 & 444 & 5 \\
\hline 1476 & 6 & 1632 & 7 & 672 & 7 & 668 & 6 \\
\hline 1153 & 3 & 1216 & 3 & 220 & 3 & 308 & 3 \\
\hline
\end{tabular}

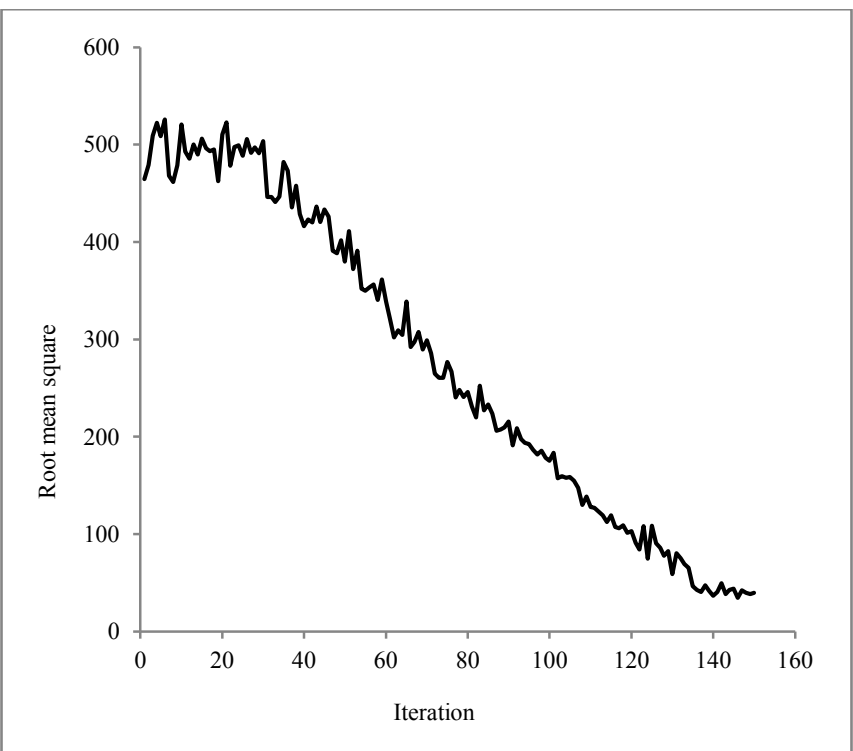

Figure 4. Objective function for the second experiment.

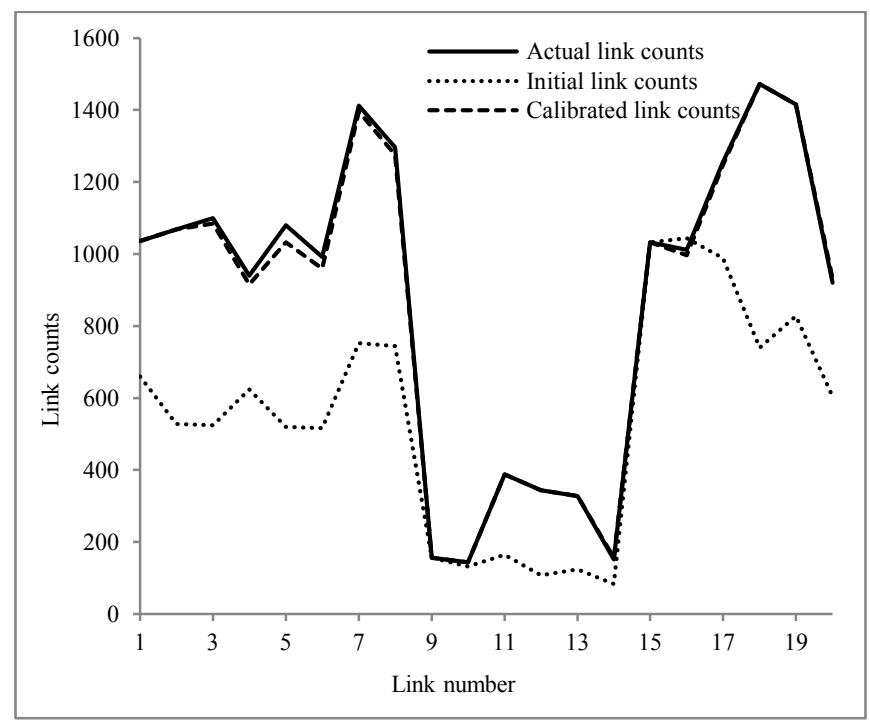

Figure 5. Links counts for the first time-period of the second experiment.

Similar to Fig. 5 and 6, Fig. 7 - Fig 11, show the link counts and GEH statistics results for all links in the network for the second, tird, and fourth time period of the simulation. The calibrated results are significantly closer to the actual values, relative to the uncalibrated results. In adition, all links have a GEH statistic below the threshold limit of 5 .
Table IV summarizes the calibration results for the second experiment. The objective function improved from 464.65 to 34.34. The initial difference between actual and simulated link counts was $40.72 \%$. After calibration, this difference was $1.04 \%$. The GEH statistic for the calibrated model was less than 5 for $100 \%$ of the links.

TABLE IV. SUMmARY OF CALIBRATION RESUltS FOR THE SECOND EXPERIMENT

\begin{tabular}{|l|c|c|c|}
\hline & RMS & Total Volumes & GEH \\
\hline Initial & 464.65 & 44396 & $<5$ for $12.5 \%$ of the \\
links
\end{tabular}

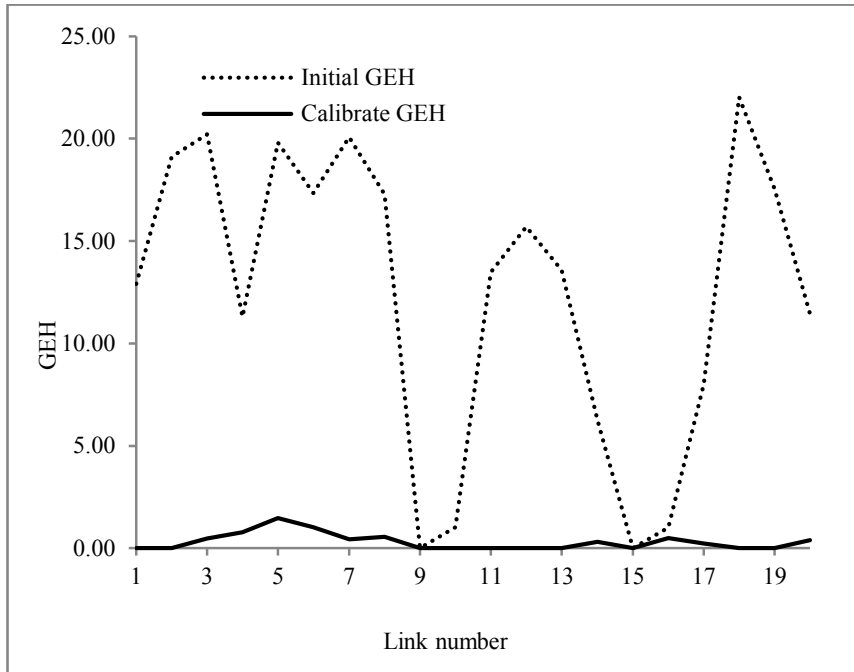

Figure 6. GEH statistic for the first time period of the second experiment.

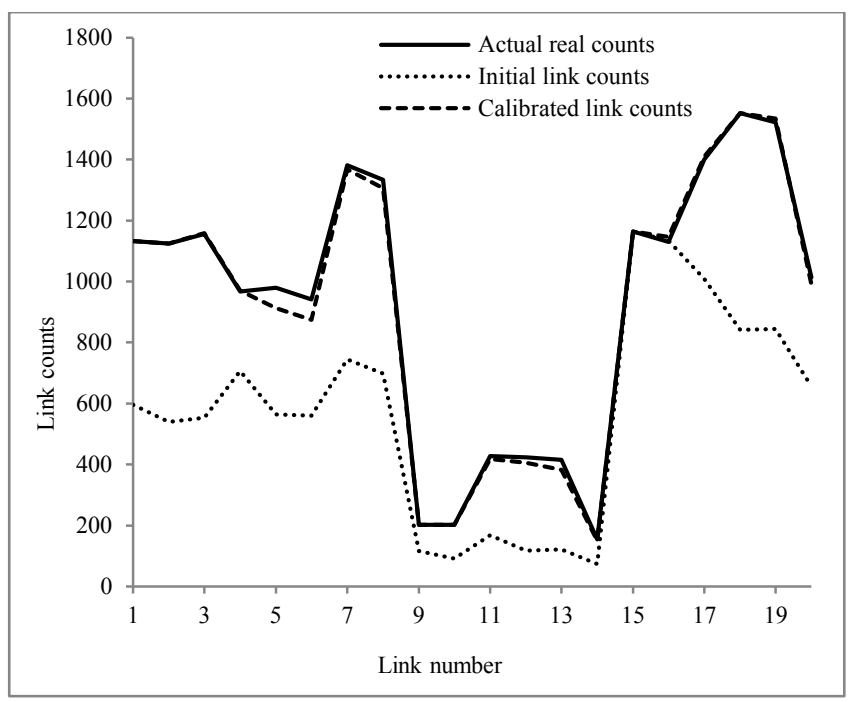

Figure 7. Links counts for the second time period of the second experiment.

In this experiment, optimal model parameters are determined in order to reproduce time-dependent link counts. The calibrated parameters take a single value during the entire simulation process. That is, they are not time- 
dependent. In contrast, the link counts are time-dependent. These results illustrate the ability of the proposed calibration methodology to adjust model parameters so as to calibrate the time-dependent link counts.

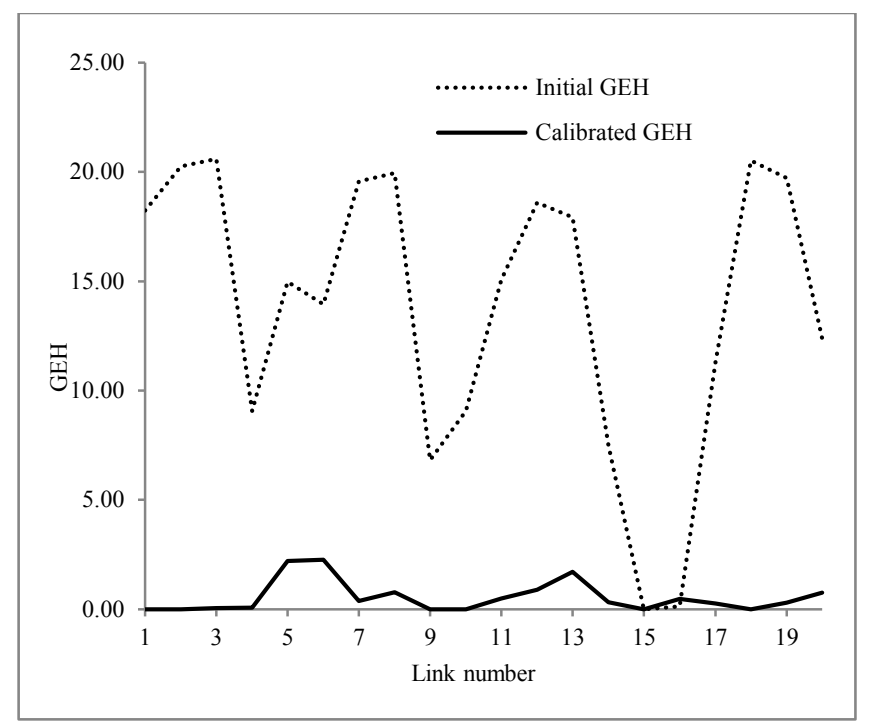

Figure 8. GEH statistic for the second time period of the second experiment.

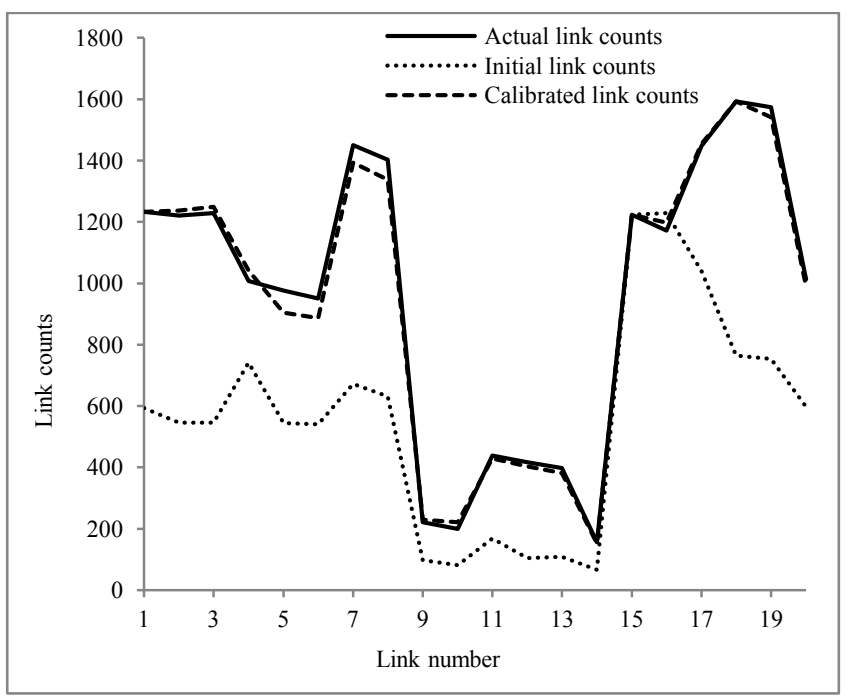

Figure 9. Links counts for the third time period of the second experiment.

\section{CONCLUSION}

The calibration methodology proposed in this study was able to calibrate time-dependent link counts of a microscopic traffic flow simulation model. The methodology relies on a SPSA algorithm to determine the calibrated set of model parameters. The experimental results illustrate the effectiveness of the proposed methodology. Two different networks were calibrated. One network was calibrated for a single simulation time period, and the other was calibrated for four simulation time periods. In both cases, all calibration guidelines were satisfied. The proposed methodology minimizes the difference between actual and simulated time-dependent link counts by considering simultaneously all model parameters. The proposed calibration methodology is being developed independently of the characteristics of any particular microscopic traffic flow simulation model. Currently, it is being tested using CORSIM models; further research will test the methodology using various microscopic traffic flow simulation models.

This research will expand the methodology to simultaneously consider link counts, speeds, and associated bottlenecks. Furthermore, simultaneous calibration of FRESIM (freeways) and NETSIM (surface streets) models is currently under development. In addition, a bi-level optimization framework is currently under development to enable the determination of an optimal and more efficient set of coefficients for the gain functions used by the SPSA and the corresponding set of calibrated microsimulation model parameters.

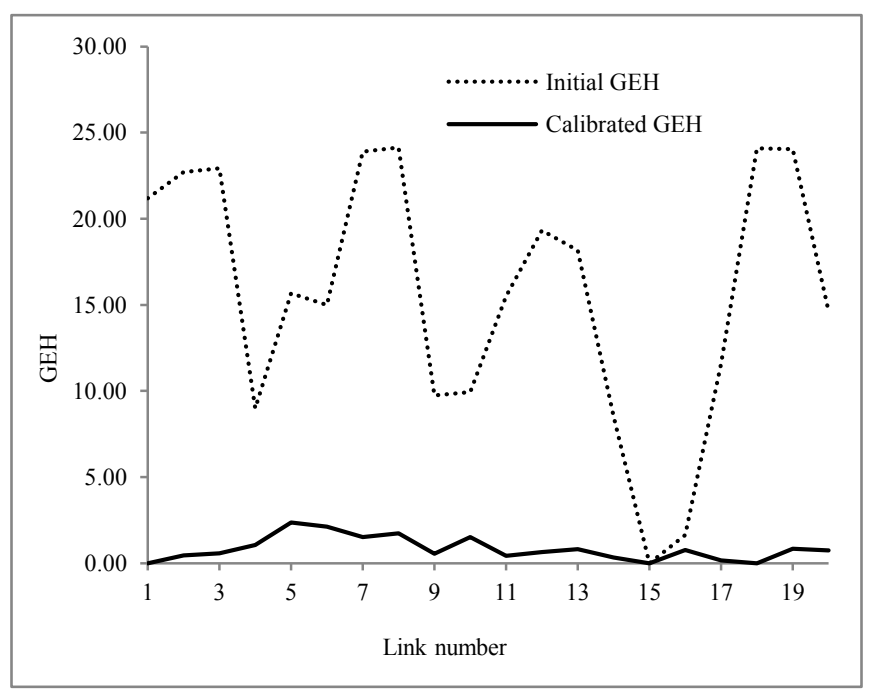

Figure 10. GEH statistic for the third time period of the second experiment.

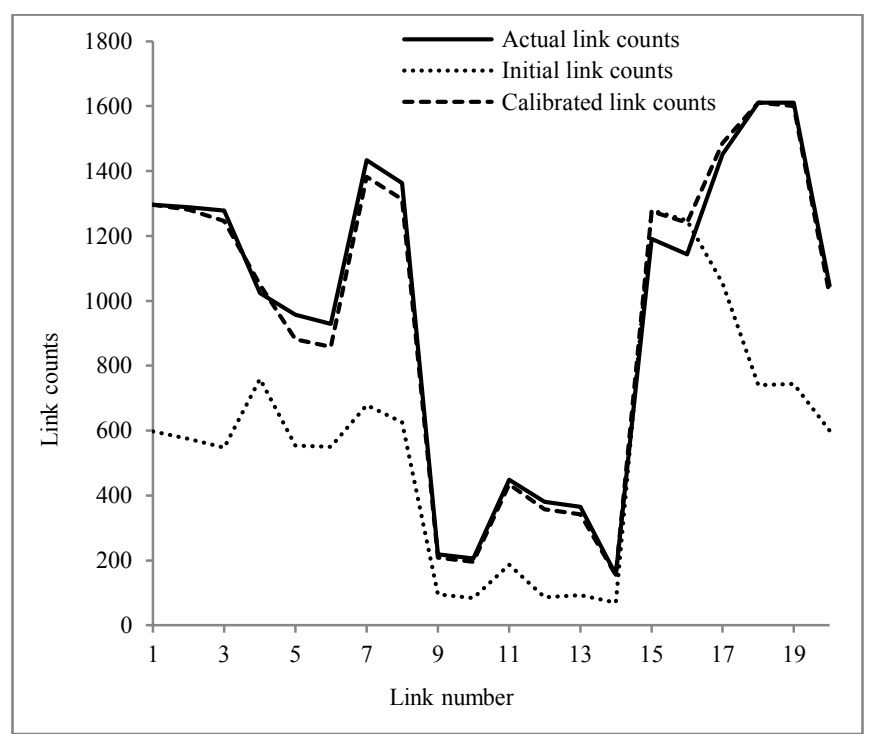

Figure 11. Links counts for the fourth time period of the second experiment. 


\section{REFERENCES}

[1] S. Kim and R. F. Benekohal, "Comparison of control delays from CORSIM and the highway capacity manual for oversaturated signalized intersections," J. Transp. Eng. 131(12), pp. 917-923, 2005.

[2] L. E. Owen, Y. Zhang, L. Rao and G. McHale, "Traffic flow simulation using CORSIM (Presented Conference Paper style)," Presented at 2000 Winter Simulation Proceedings, Orlando FL, December 10-13, 2000, 1143 -1147 vol.2.

[3] A. Kondyli, I. Soria, A. Duret and L. Elefteriadou, "Sensitivity analysis of CORSIM with respect to the process of freeway flow breakdown at bottleneck locations," Simulation Modelling Practice and Theory 22(0), pp. 197-206, 2012.

[4] P. Holm, D. Tomich, J. Sloboden and C. Lowrance, "Traffic analysis toolbox volume IV: Guidelines for applying CORSIM microsimulation modeling software," ITT Industries, Inc., Tech. Rep. FHWAHOP-07-079, 2007.

[5] K. Kim and L. R. Rilett, "Simplex-based calibration of traffic microsimulation models with intelligent transportation systems data," 2003.

[6] A. L. Cunha, J. E. Bessa Jr. and J. R. Setti, "Genetic algorithm for the calibration of vehicle performance models of microscopic traffic simulators (Presented Conference Paper style)," Presented at 14th Portuguese Conference on Artificial Intelligence, Aveiro Portugal, EPIA 2009, October 12-15, 2009, ISBN: 978-3-64204685-8.

[7] J. Kim, B, (. Park, J. Lee and J. Won. "Determining optimal sensor locations in freeway using genetic algorithm-based optimization," Eng Appl Artif Intell 24(2), pp. 318-324, 2011.

[8] A. Stevanovic, P. T. Martin and J. Stevanovic,"VisSimbased genetic algorithm optimization of signal timings," Transp. Res. Rec. (2035), pp. 59-68, 2007.

[9] B. (. Park, I. Yun and K. Ahn, "Stochastic optimization for sustainable traffic signal control," Int. J. Sustain. Transp. 3(4), pp. 263-284, 2009.

[10] B. (. Park and A. Kamarajugadda, "Development and evaluation of a stochastic traffic signal optimization method," Int. J. Sustain. Transp. 1(3), pp. 193-207, 2007.

[11] G. G. Schultz and L. R. Rilett, "Calibration of distributions of commercial motor vehicles in CORSIM," Transp. Res. Rec. (1934), pp. 246-255, 2005.

[12] S. I. Chien and J. Luo, "Optimization of dynamic ramp metering control with simultaneous perturbation stochastic approximation," Control Intell. Syst. 36(1), pp. 57-63, 2008.

[13] J. C. Spall, "Stochastic optimization and the simultaneous perturbation method (Presented Conference Paper style)," Presented at 1999 Winter Simulation Conference Proceedings (WSC), Phoenix AZ, December 5-8, 1999, ISBN:0-7803-5780-9.
[14] J. C. Spall, "Introduction to Stochastic Search and Optimization : Estimation, Simulation, and Control," 2003.

[15] R. Balakrishna, C. Antoniou, M. Ben-Akiva, H. N. Koutsopoulos and Y. Wen, "Calibration of microscopic traffic simulation models: Methods and application," Transp. Res. Rec. (1999), pp. 198-207, 2007.

[16] J. Lee, "Calibration of traffic simulation models using simultaneous perturbation stochastic approximation (SPSA) method extended through bayesian sampling methodology," ProQuest Dissertations and Theses, 2008.

[17] J. Ma, H. Dong and H. M. Zhang, "Calibration of microsimulation with heuristic optimization methods," Transp. Res. Rec. (1999), pp. 208-217, 2007.

[18] E. E. Ozguven and K. Ozbay, "Simultaneous perturbation stochastic approximation algorithm for solving stochastic problems of transportation network analysis: Performance evaluation," Transp. Res. Rec. (2085), pp. 12-20, 2008.

[19] J. Yuan, S. H. Ng and K. L. Tsui, "Application of stochastic approximation methods for stochastic computer models calibration (Presented Conference Paper style)," Presented at IEEE International Conference on Industrial Engineering and Engineering Management, IEEM2010, Macau, December 7-10 2010, $1606-1610$.

[20] J. J. Schneider, S. Kirkpatrick and Springer, "Stochastic Optimization," 2006.

[21] J. L. Maryak and J. C. Spall, "Simultaneous perturbation optimization for efficient image restoration. Aerospace and Electronic Systems," IEEE Transactions on 41(1), pp. 356-361, 2005.

[22] E. Brockfeld, R. D. Kuhne and P. Wagner, "Calibration and validation of microscopic models of traffic flow," Transp. Res. Rec. (1934), pp. 179-187, 2005.

[23] J. P. McCarthy, "Traffic analysis toolbox," Public Roads 68(5), pp. 52-56, 2005.

[24] D. Breski, D. Cvitanic and I. Lovric, "Sensitivity analysis of the CORSIM simulation model parameters; analiza osjetljivosti parametara simulacijskog modela CORSIM," Gradevinar 58(7), pp. 539-548, 2006.

[25] K. Kim and L. R. Rilett, "Calibration of microsimulation supply and demand parameters using bi-level algorithm (Presented Conference Paper style)," Presented at Proceedings of the Seventh International Conference on: Applications of Advanced Technology in Transportation, Boston MA, August 5-7, 2002, 902909.

[26] T. Toledo, M. Ben-Akiva, D. Darda, M. Jha and H. N. Koutsopoulos, "Calibration of microscopic traffic simulation models with aggregate data," 2004 . 